\title{
A narrative review of primary spontaneous pneumomediastinum: a poorly understood and resource-intensive problem
}

\author{
Clinton T. Morgan ${ }^{1}$, James D. Maloney ${ }^{2}$, Malcolm M. Decamp ${ }^{2}$, Daniel P. McCarthy ${ }^{2}$ \\ ${ }^{1}$ Division of Cardiothoracic Surgery, Department of Surgery, University of Kentucky, Lexington, KY, USA; ${ }^{2}$ Division of Cardiothoracic Surgery, \\ Clinics Department of Surgery, University of Wisconsin Hospitals, Madison WI, USA \\ Contributions: (I) Conception and design: CT Morgan, JD Maloney; (II) Administrative support: None; (III) Provision of study materials or patients: \\ None; (IV) Collection and assembly of data: CT Morgan; (V) Data analysis and interpretation: All authors; (VI) Manuscript writing: All authors; (VII) \\ Final approval of manuscript: All authors. \\ Correspondence to: Clinton T. Morgan, MD, PHD. Division of Cardiothoracic Surgery, Department of Surgery, University of Kentucky 740 S. \\ Limestone, Suite A301, Lexington, KY 40536, USA. Email: clinton.morgan@uky.edu.
}

\begin{abstract}
Primary spontaneous pneumomediastinum (PSPM) is a benign self-limited condition that can be difficult to discriminate from esophageal perforation. This may trigger costly work-up, transfers and hospital admissions. To better understand this diagnostic dilemma and current management, we undertook the most comprehensive and up to date review of PSPM. The PubMed database was searched using the MeSH term "Mediastinal Emphysema"[Mesh], to identify randomized controlled trials, meta-analyses and case series (including 10 or more patients) relevant to the clinical presentation and management of patients with PSPM. There were no relevant randomized controlled trials or meta-analyses. Nineteen case series met our criteria, including a total of 535 patients. The average mean age was 23 years with a 3:1 male predominance. Chest pain was the most common symptom, found in $70.9 \%$ of the patients. Dyspnea and neck pain were the second and third most common symptoms, found in $43.4 \%$ and $32 \%$ of the patients, respectively. Subcutaneous emphysema was the most common sign (54.2\%). Common histories included smoking (29.6\%), cough $(27.7 \%)$, asthma (25.9\%), physical exertion (21.1\%) and recent retching or emesis (13\%). Nearly all patients $(96.9 \%)$ underwent chest X-ray (CXR). Other diagnostic studies included computed tomography $(65 \%)$ and esophagram (35.6\%). Invasive studies were common, with $13 \%$ of patients undergoing esophagogastroduodenoscopy and $14.6 \%$ undergoing bronchoscopy. The rate of hospital admission was $86.5 \%$, with an average length of stay of 4.4 days. No deaths were reported. Notably, we identified a dearth of information regarding the vitals, laboratory values and imaging findings specific to patients presenting with PSPM. We conclude that PSPM is a benign clinical entity that continues to present a resource-intensive diagnostic challenge and that data on the vitals, labs, and imaging findings specific to PSPM patients is scant. An improved understanding of these factors may lead to more efficient diagnosis and management of these patients.
\end{abstract}

Keywords: Spontaneous pneumomediastinum; esophageal perforation; Boerhaave's

Submitted Jan 29, 2021. Accepted for publication Apr 09, 2021.

doi: $10.21037 /$ jtd-21-193

View this article at: http://dx.doi.org/10.21037/jtd-21-193

\section{Introduction}

Primary spontaneous pneumomediastinum (PSPM) is a self-limited condition that is problematic due to the difficulty in discriminating it from life-threatening processes such as esophageal perforation and mediastinitis.
PSPM patients present with mediastinal emphysema and no traumatic or iatrogenic cause. By contrast, secondary pneumomediastinum may be secondary to processes such as esophageal perforation and traumatic injury of the esophagus or tracheobronchial tree. Hamman first 
described PSPM in 1939 (1). The associated auscultation finding of "crunching" with cardiac contraction is referred to as the "Hamman sign." Despite its moniker, PSPM can often be associated with processes causing sudden intrathoracic pressure changes. For example, these changes may be associated with coughing or retching. Patients frequently have a history of asthma or smoking (2). Pathophysiologically, the condition is thought to result from pressure changes leading to alveolar rupture followed by tracking of air along the bronchovascular tissue plane, a process known as the Macklin effect (3). Cases have attributed PSPM to other Valsalva events, such as child-birth (4), diabetic ketoacidosis (5), fellatio (6) and cannabinoid hyperemesis syndrome (7). However, PSPM patients may present without any clear inciting event or predisposing factors $(8,9)$.

Patients with PSPM frequently present with chest pain and crepitus, prompting hospital admission and an extensive workup to rule out esophageal perforation. An improved understanding of the typical presentation and history of patients with PSPM may help discriminate these patients from those with more life-threatening conditions.

The aims of this study were to better characterize contemporary diagnosis and management of this condition based on the best clinical evidence available, which is limited to retrospective case series. We undertook a comprehensive review of PSPM case series to evaluate demographics, symptoms, clinical signs, histories, vitals, diagnostic methods, management, treatment and recurrence rates. We present the following article in accordance with the Narrative Review reporting checklist (available at http:// dx.doi.org/10.21037/jtd-21-193).

\section{Methods}

We conducted a PubMed search using the MeSH term "Mediastinal Emphysema"[Mesh], to identify randomized controlled trials, meta-analyses and case series (with 10 or more patients) relevant to the clinical presentation and management of patients with PSPM. This search strategy, conducted in May 2020, yielded 3,547 results. All titles and abstracts were reviewed. There were no relevant randomized controlled trials or meta-analyses. There were multiple small case series. Of these, we selected case series with more than 10 patients and excluded nonEnglish language studies. Fourteen case series met our initial criteria. One series was removed because it included secondary pneumomediastinum cases. Evaluation of the remaining thirteen citations resulted in identification of an additional six case series that were appropriate for inclusion. Clinical variables were abstracted from the texts and included: demographics: age and gender; symptoms: chest pain, dyspnea, and neck pain; clinical signs: subcutaneous emphysema, Hamman sign, and pneumothorax; histories: smoking, cough, asthma, physical exertion, and retching/ emesis; vitals: fever, tachycardia, hypotension, tachypnea, and hypoxemia; diagnostic studies: chest X-ray (CXR), chest computed tomography (CT), esophagram, EGD, and bronchoscopy; management/treatment: antibiotics, thoracostomy tubes for concurrent pneumothorax, hospital admission and duration; and recurrence rates.

\section{Results}

\section{Demographics}

Nineteen case series were appropriate for inclusion (2,8,9,10-25). Publication dates ranged from 1991 to 2017. On average the studies included a relatively small number (28.2) of patients (Table 1). In total, the combined case series included 535 patients with PSPM. Of the fifteen studies that reported mean ages, the average mean age was 23 years. All but two studies included pediatric patients. There was a clear male predominance ( $72 \%$ male versus $28 \%$ female), which has been noted previously (26).

\section{Clinical bistory}

Most of the studies reported on possible inciting or predisposing factors in patients presenting with PSPM. Ten of the studies reported smoking history, with an average rate of $29.6 \%$ (Table 2). The incidence of cough averaged $27.7 \%$ (Table 2). A history of asthma was noted in fifteen of the studies, with an average rate of $25.9 \%$ (Table 2). Recent physical exertion was reported in $21.1 \%$ (Table 2). Finally, retching/emesis was reported in $13 \%$ (Table 2). Taken together, these findings bolster the conclusion that processes involving sudden intrathoracic pressure changes are strongly associated with PSPM.

\section{Symptoms and signs}

Chest pain was the most common symptom and was the only symptom reported in each of the nineteen case series (Table 3). Taken together, $70.9 \%$ of patients with PSPM presented with chest pain. Dyspnea was the second most 
Table 1 Primary spontaneous pneumomediastinum demographics

\begin{tabular}{|c|c|c|c|c|c|}
\hline Study & Mean age & Age range & $\mathrm{N}$ & Female & Male \\
\hline Kaneki et al. 2000 & NR & $13-27$ & 33 & 7 & 26 \\
\hline Gerazounis et al. 2003 & NR & $12-32$ & 22 & 4 & 18 \\
\hline Jougon et al. 2003 & 25 & $16-46$ & 12 & 1 & 11 \\
\hline Weissberg \& Weissberg 2004 & NR & $15-37$ & 22 & 10 & 12 \\
\hline Campillo-Soto et al. 2005 & 36.8 & $11-90$ & 36 & 11 & 25 \\
\hline Newcomb \& Clarke 2005 & NR & $11-58$ & 18 & 4 & 14 \\
\hline Freixinet et al. 2005 & 21.4 & $14-36$ & 32 & 8 & 24 \\
\hline Caceres et al. 2008 & 27 & $3-71$ & 28 & 12 & 16 \\
\hline Al-Mufarrej et al. 2008 & 25.5 & $19-39$ & 17 & 6 & 11 \\
\hline Takada et al. 2008 & 20.1 & $13-28$ & 25 & 7 & 18 \\
\hline Perna et al. 2010 & 27.3 & $16-42$ & 47 & 14 & 33 \\
\hline Ryoo 2012 & 18.3 & $10-38$ & 32 & 7 & 25 \\
\hline Okada et al. 2014 & 22 & $13-41$ & 20 & 1 & 19 \\
\hline Bakhos et al. 2014 & 19 & $5-57$ & 49 & 23 & 26 \\
\hline Ebina et al. 2017 & 19.7 & $5-36$ & 34 & 8 & 26 \\
\hline
\end{tabular}

reported symptom with an average rate of $43.4 \%$ (Table 3). Finally, 32.9\% of patients reported neck pain (Table 3). Subcutaneous emphysema was the most common sign with an average rate of $54.2 \%$ (Table 4 ). The average rate of Hamman sign in the eleven studies reporting it was $23.5 \%$ (Table 4). Finally, $10.8 \%$ of patients presenting with PSPM were also found to have a pneumothorax (Table 4). Taken together, these results present a clearer picture of the common presenting symptoms and signs of patients with PSPM.

\section{Observations}

We found limited data on the presenting vitals and laboratory values for patients with PSPM. For example, the presence of fever was reported in only six studies, with an average rate of $18.8 \%$ (Table S1). The absence of tachycardia was noted by Jougon et al. (13) while Campillo-Soto et al. (15) and Weissberg \& Weissberg (8) reported tachycardia in $5.6 \%$ and $7 \%$ of the patients, respectively (Table S1). Jougon et al. (13) was the only report commenting on hypotension, which was not observed in their case series. One patient with tachypnea was reported in the case series by Campillo-Soto et al. (15) and one patient with hypoxemia was noted in the case series by Mondello et al. (18), respectively (Table S1). Finally, only seven of the studies reported on the presence of leukocytosis, with an average rate of $30.8 \%$, and four studies reported white blood cell count (Table S2). Taken together, data on the typical vitals and basic laboratory values for 
Table 2 Histories associated with primary spontaneous pneumomediastinum

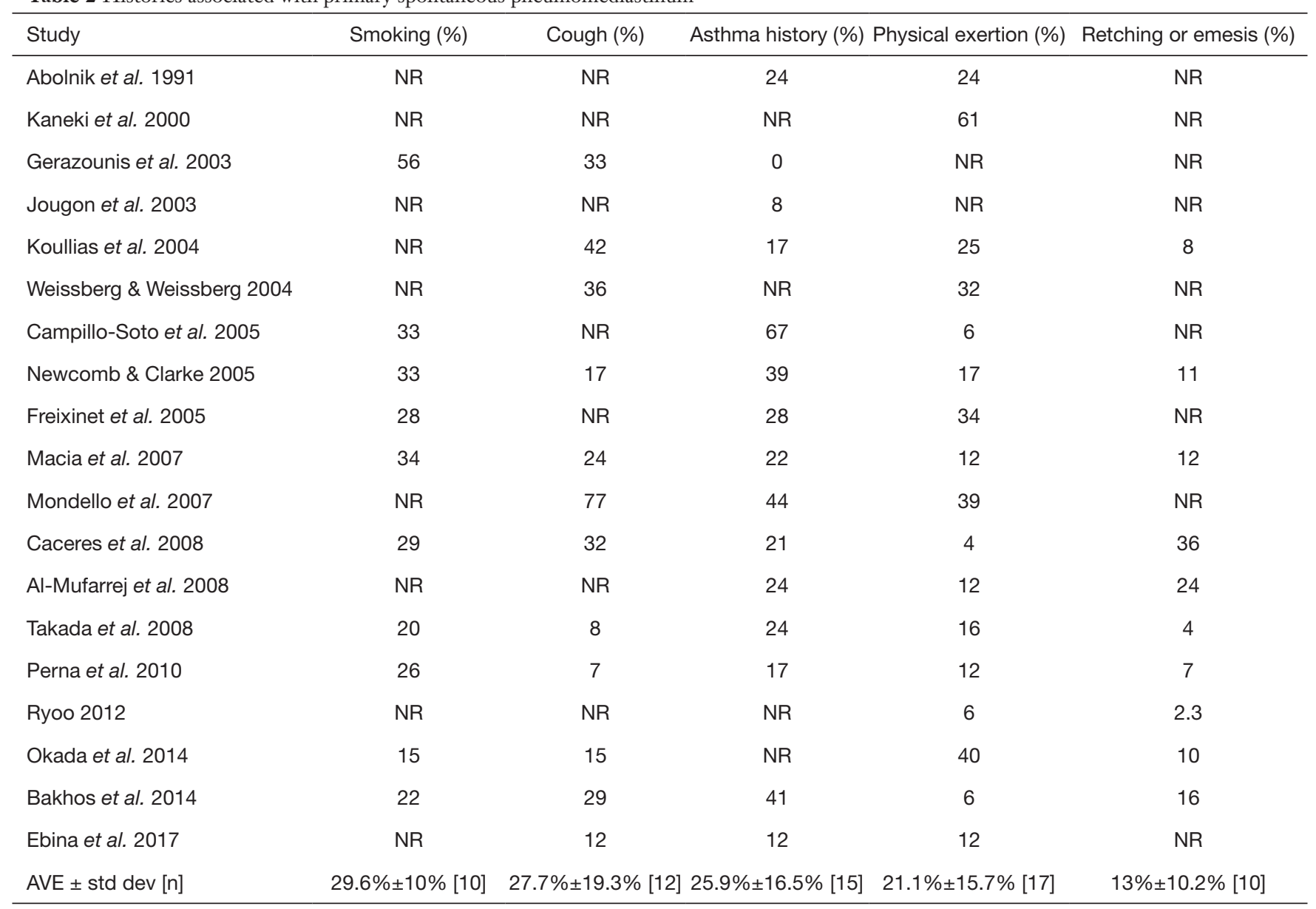

patients presenting with PSPM is scant.

\section{Diagnostic methods}

Which diagnostic studies are typically performed in the evaluation of spontaneous pneumomediastinum? CXR was obtained in $96.9 \%$ of patients (Table 5). CT scans were obtained in $65 \%$ (Table 5). It was not possible to determine if CT imaging was required for diagnosis of pneumomediastinum or obtained to rule in/out other potential disease processes. In the series reported by Kaneki et al., they obtained CT in all patients to better understand the CT findings of PSPM (11). They concluded that approximately $30 \%$ of PSPM cases were poorly detected by CXR alone and that PSPM is likely an underdiagnosed entity. Three of the studies specifically commented on the presence or absence of effusion on imaging. None of the patients ( $\mathrm{n}=104$ patients) in these series had an effusion
(Table S3). In the case series reported by Bakhos et al., comparison was made with a group of thirteen esophageal perforation patients (24). Notably, the rate of effusion in the esophageal perforation group was $31 \%$. Esophagrams were reported in fifteen studies, with a rate of $35.6 \%$ of patients (Table 5). Finally, EGDs were performed in $13 \%$ of the patients (Table 5). Bronchoscopy was performed in 14.6\% of patients (Table 5). These results show that CXR is the most common diagnostic modality used in the diagnosis of pneumomediastinum, but CT imaging and esophagrams are common diagnostic adjuncts.

\section{Management and treatment}

Interventions were rare. Furthermore, they were almost exclusively limited to complications arising from concurrent pneumothorax. Overall, we found scant data regarding management of concurrent pneumothorax, but several case 
Table 3 Symptoms of primary spontaneous pneumomediastinum

\begin{tabular}{|c|c|c|c|}
\hline Study & Chest pain (\%) & Dyspnea (\%) & Neck pain (\%) \\
\hline Kaneki et al. 2000 & 100 & 58 & 70 \\
\hline Gerazounis et al. 2003 & 89 & 59 & NR \\
\hline Jougon et al. 2003 & 50 & NR & 25 \\
\hline Weissberg \& Weissberg 2004 & 82 & 45 & NR \\
\hline Campillo-Soto et al. 2005 & 27 & 22 & 14 \\
\hline Newcomb \& Clarke 2005 & 89 & 67 & 11 \\
\hline Freixinet et al. 2005 & 78 & 41 & NR \\
\hline Caceres et al. 2008 & 54 & 39 & 44 \\
\hline Al-Mufarrej et al. 2008 & 59 & 41 & 12 \\
\hline Takada et al. 2008 & 68 & 44 & 52 \\
\hline Perna et al. 2010 & 60 & 26 & 38 \\
\hline Ryoo 2012 & 51 & 14 & 23 \\
\hline Okada et al. 2014 & 75 & 40 & 10 \\
\hline Bakhos et al. 2014 & 65 & 51 & 29 \\
\hline Ebina et al. 2017 & 61 & 29 & 29 \\
\hline
\end{tabular}

series did report thoracostomy tube placement with a rate of $76 \%$ (Table S4). A single patient, out of the entire 535 patients in the nineteen series, underwent an operation for "tension pneumothorax" within several hours of presentation. The patient underwent a thoracotomy with opening of the mediastinal pleura (22). To our knowledge, this is the only patient who underwent an operation and the indication was tension pneumothorax. The only other intervention noted was drainage of subcutaneous emphysema in 10 of the 18 total patients reported in the series of Mondello et al. (18). This may be more reflective of institutional or individual surgeon practice and does not appear to be a common treatment regimen. Taken together, the need for invasive interventions in PSPM patients is rare and almost exclusively undertaken for management of concurrent pneumothorax.

Despite the self-limited nature of PSPM, these patients are frequently admitted to the hospital and treated with antibiotics. Six studies reported on the use of antibiotics with $43 \%$ of patients receiving antibiotics (Table 6). Importantly, there are no reported cases of mediastinitis and no prospective studies on the use of antibiotics in PSPM. There were no reported mortalities. Fifteen case series reported rates of PSPM recurrence, with an average rate of $0.98 \%$ (Table S5). The follow up periods varied widely, as did the methods of follow up (Table S5). We did not find data regarding the rate of hospital transfers. $86.5 \%$ of the patients were admitted to the hospital with a mean length of hospital stay of 4.4 days (Table 6). Due to the retrospective nature of this case series review, the current available data do not have the granularity to determine reasons (e.g., pain management, diagnostic uncertainty, etc.) for admission or length of stay. Together these data show that the vast majority of patients with this benign condition are admitted to the hospital for multiple days and nearly half of them are treated with antibiotics. 
Table 4 Signs of primary spontaneous pneumomediastinum

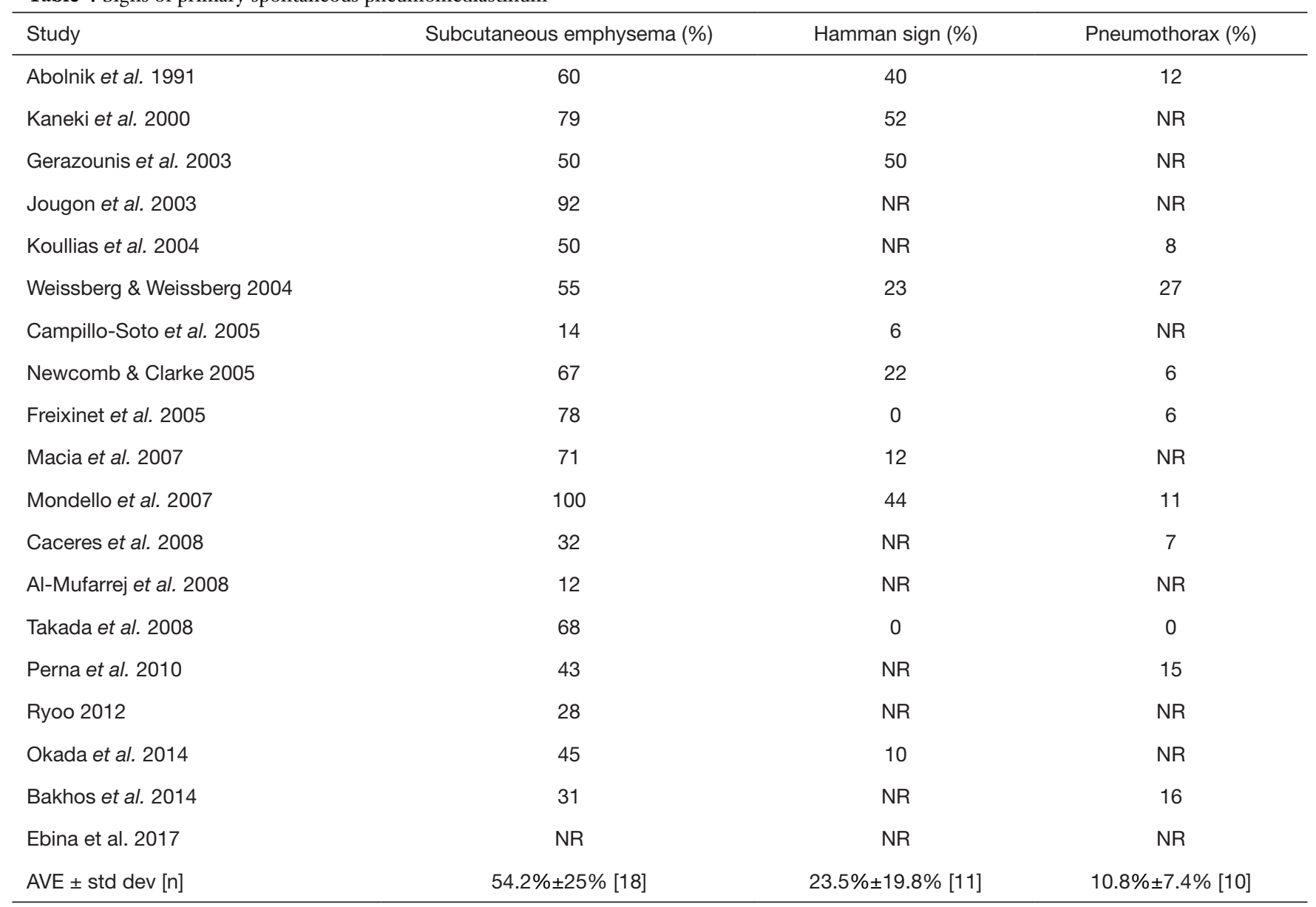

\section{Discussion}

PSPM continues to pose a difficult clinical problem because it can be difficult to discriminate from life-threatening conditions with a similar presentation, namely esophageal perforation. While this difficulty is a defining feature encountered by practicing clinicians, the reasons for this difficulty are unclear. We sought to understand the reasons for this diagnostic difficulty. Case series represent the best evidence currently available. Our review of these case series has the expected limitations associated with retrospective studies, including the possibility of selection and publication bias. Other limitations include the potential for variable definitions of clinical parameters on the case series level.

Although the clinical presentation of esophageal perforation is beyond the scope of this review, it is clear that we need to better understand how it differs from that of PSPM. Chest pain, dyspnea and subcutaneous emphysema are common in the presentation of PSPM, but they are also common in the presentation esophageal perforation (27). In their systematic review of 33 case series including 1,452 esophageal perforation patients, Hasimoto et al. found the mean age was 55.2 years (27). Approximately $80 \%$ of esophageal perforations have a clear iatrogenic or traumatic/ post-surgical etiology in their history. Spontaneous esophageal perforations, i.e., Boerhaave's syndrome, represented approximately 20 percent of the cases (27). It is this patient population that must be discriminated from PSPM patients. The typical Boerhaave's patient is a $~ 55-65$ years old male, often with a history of excessive alcohol and/ or food consumption following by severe retching/emesis. While age is an important demographic variable that differs between PSPM and esophageal perforation, Boerhaave's cannot be excluded from the differential diagnosis simply based on patient age.

Our review reveals a very limited understanding of the 
Table 5 Diagnostic studies

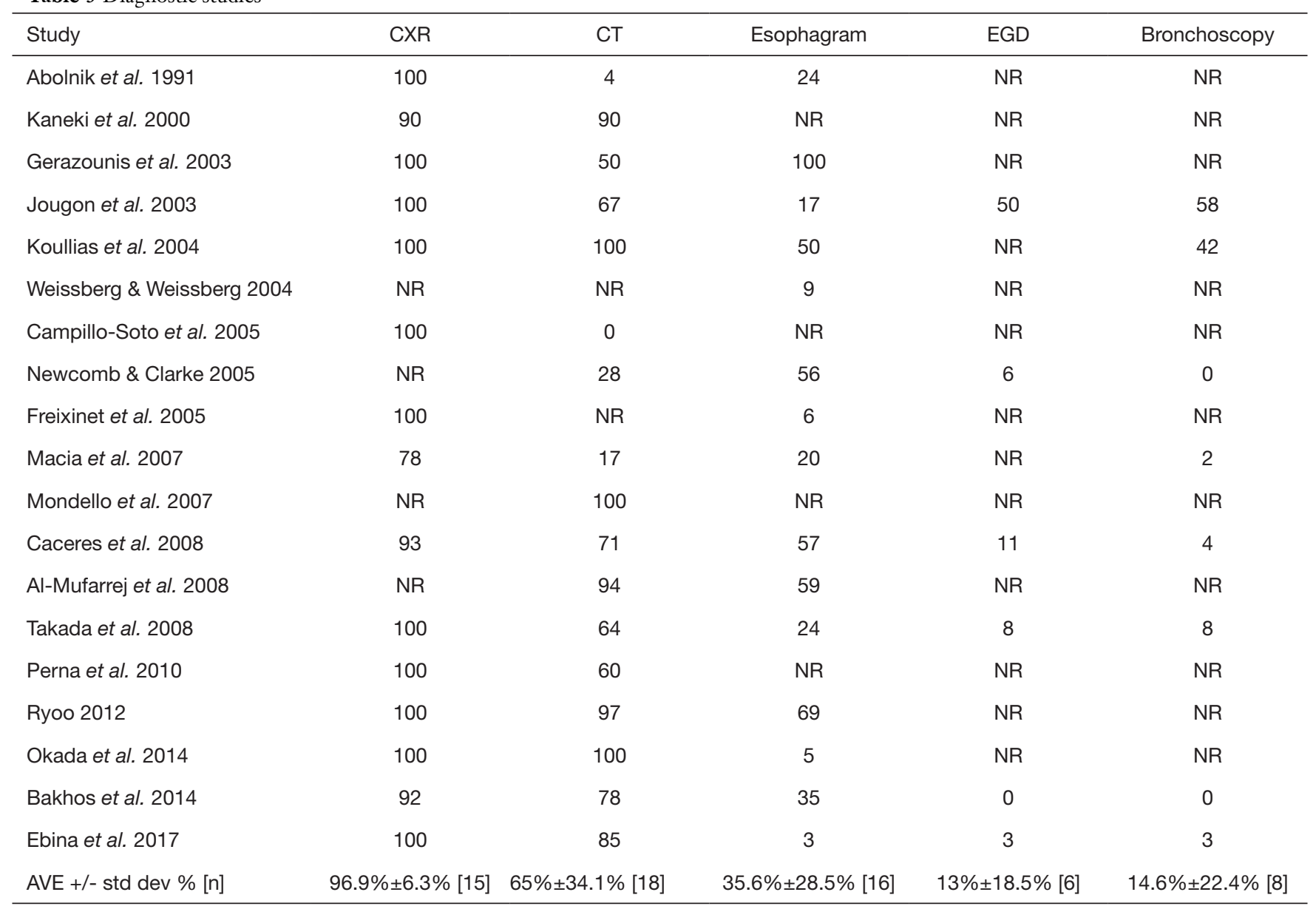

vitals and basic laboratory values of patients presenting with PSPM. Indeed, there is insufficient data to make any meaningful conclusions regarding the rates of tachycardia, hypotension, tachypnea or hypoxemia in this patient population. We suspect that the presence of fever and leukocytosis in this patient population, coupled with minimal understanding of other vitals and labs, likely contributes to concern for esophageal perforation. A better understanding of these basic clinical variables may improve our ability to discriminate between patients with PSPM and patients with esophageal perforation.

CT imaging is typically inadequate to discern between PSPM and esophageal perforation. To date, there is scant radiologic data specifically comparing non-contrast CT findings between patients with PSPM and esophageal perforation. Notably, while pleural effusions are apparent in $\sim 10-30 \%$ of esophageal perforations, zero effusions were reported in our review of these PSPM case series. We anticipate that further radiologic studies comparing PSPM and esophageal perforation cases could significantly improve our ability to discriminate between these two diagnoses.

Since CXR and non-contrast CT imaging is currently insufficient to discriminate between PSPM and esophageal perforation two key questions arise: (I) which patients need studies beyond CXR or CT and (II) which study is needed? Fluoroscopic contrast esophagram has traditionally been regarded as the gold-standard for identification of esophageal perforation (28). In our experience, many referring centers do not have fluoroscopic esophagram capabilities and patients are frequently transferred solely due to lack of this resource. An important development in this area is the growing body of evidence suggesting that contrast computed tomography esophagram has a sensitivity and negative predict value for esophageal perforation that is equivalent to traditional fluoroscopic contrast esophagram (29). Because CT esophagram can be performed 
Table 6 Management of Primary Spontaneous Pneumomediastinum patients

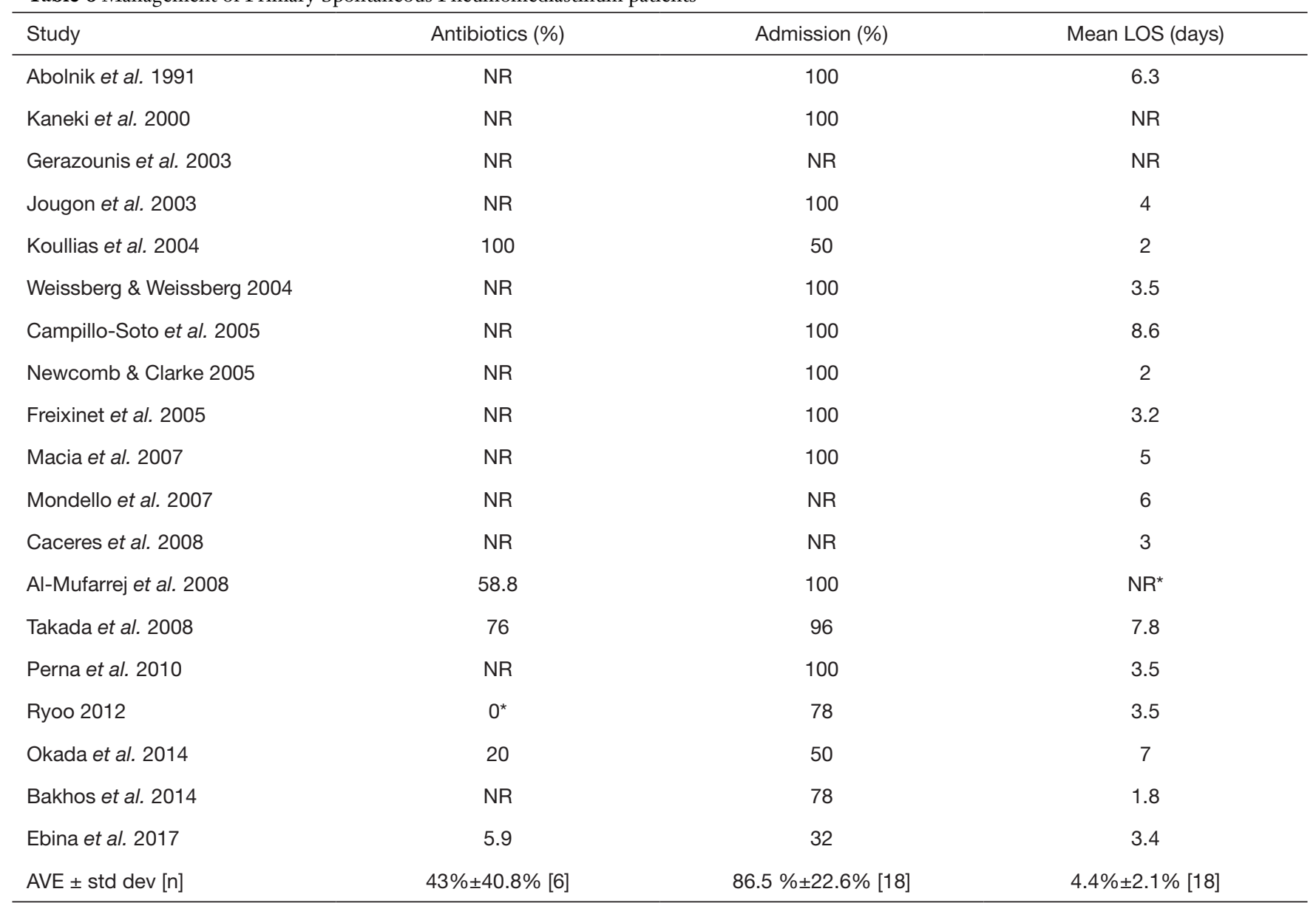

**, for patients with no other co-morbidities, the length of hospital stay ranged from 23-72 hours (average 40.5 hours). Patients admitted with significant dehydration, asthma exacerbation, or drug intoxication had longer hospital stays (5-11 days with average of 7 days).

at essentially any center with CT imaging capabilities, it has the potential to significantly decrease the interfacility transfers and diagnostic delays. Either fluoroscopic contrast esophagram or contrast CT esophagram can be used when evaluation for esophageal perforation is indicated. We use the term "contrast esophagram" to refer to either of these modalities. While there is clearly a role for contrast esophagram in the workup of select patients presenting with pneumomediastinum, currently there is no consensus regarding which pneumomediastinum patients should undergo a study. The current PSPM literature does not address this issue. Taken together, these findings suggest that a high index of suspicion, with case by case physician judgement, including communication between Emergency Medicine physicians and Thoracic Surgeons, remains important. Finally, if diagnostic uncertainty regarding possible esophageal perforation remains, contrast esophagram is indicated.

Even when the diagnosis of PSPM is clearly established, questions remain regarding optimal management. Because small concurrent pneumothoraces are present in approximately ten percent of these patients, and may be occult, thoracic CT is a valuable adjunct in these patients. Given the potential for tension physiology, we suggest that concurrent pneumothorax should be managed in a manner similar to primary spontaneous pneumothorax patients. We propose that when the diagnosis of PSPM without concurrent pneumothorax is made with confidence, no additional testing, work-up or treatment is indicated. While admission may be indicated for management of pain, nausea/emesis, or other reasons, it is likely unnecessary for stable patients who meet disposition criteria. A brief period of observation to confirm clinical stability is reasonable. Patients should be educated about the small, approximately 
$1 \%$, chance of recurrence. Finally, there is no evidence supporting the use of antibiotics in isolated PSPM and they are not indicated unless there is diagnostic uncertainty with concern for other etiologies, such as Boerhaave's, esophageal necrosis, or mediastinitis.

\section{Key points}

* PSPM is often difficult to discriminate from esophageal perforation due to a shared constellation of clinical signs and symptoms.

* PSPM patients typically healthy and present in their 20s whereas esophageal perforation patients typically present in their 50-60s.

* We have a very limited understanding of PSPM vitals and labs. This likely contributes to the diagnostic uncertainty.

* Imaging by CXR and CT is typically not able to discriminate between PSPM and esophageal perforation, but pleural effusion should raise concern for perforation.

* Fluoroscopic esophagrams or CT contrast esophagrams are the best tests to identify esophageal perforations, but further research is needed to determine which patients need these studies.

- Approximately $10 \%$ of PSPM patients present with concurrent pneumothorax, which should be managed by standard spontaneous pneumothorax algorithms due to the possibility of tension pneumothorax.

* An improved understanding of the above issues may significantly improve our ability to efficiently arrive at the correct diagnosis and management for these patients.

\section{Acknowledgments}

The authors thank Dr. Erik Lewis for critical reading of the manuscript.

Funding: None.

\section{Footnote}

Reporting Checklist: The authors have completed the Narrative Review reporting checklist. Available at http:// dx.doi.org/10.21037/jtd-21-193

Conflicts of Interest: All authors have completed the ICMJE uniform disclosure form (available at http://dx.doi. org/10.21037/jtd-21-193). The authors have no conflicts of interest to declare.

Ethical Statement: The authors are accountable for all aspects of the work in ensuring that questions related to the accuracy or integrity of any part of the work are appropriately investigated and resolved.

Open Access Statement: This is an Open Access article distributed in accordance with the Creative Commons Attribution-NonCommercial-NoDerivs 4.0 International License (CC BY-NC-ND 4.0), which permits the noncommercial replication and distribution of the article with the strict proviso that no changes or edits are made and the original work is properly cited (including links to both the formal publication through the relevant DOI and the license). See: https://creativecommons.org/licenses/by-nc-nd/4.0/.

\section{References}

1. Hamman L. Spontaneous mediastinal emphysema. Bull Johns Hopkins Hosp 1939;64:1-21.

2. Newcomb AE, Clarke CP. Spontaneous pneumomediastinum: a benign curiosity or a significant problem? Chest 2005;128:3298-302.

3. Macklin MT and Macklin CC. Malignant interstitial emphysema of lungs and mediastinum as an important occult complication in many respiratory diseases and other conditions: an interpretation of the clinical literature in the light of laboratory experiment. Medicine (Baltimore) 1944:23:281-358.

4. Jakes AD, Kunde K, Banerjee A. Case report: Postpartum pneumomediastinum and subcutaneous emphysema. Obstet Med 2019;12:143-5.

5. Zhang W, Chen J, Wu X, Chen L, Wei J, Xue M, Liang Q. Analysing the Clinical Features of Pneumomediastinum Associated with Diabetic Ketoacidosis in 79 Cases. Diabetes Metab Syndr Obes 2020;13:405-12.

6. Russell DW, Watts JR Jr, Powers TA. Searching for the Source of the Leak: PIE and the Macklin Effect. Ann Am Thorac Soc 2018;15:1354-6.

7. Davis W, Frye K, Shah D, et al. Cannabinoid Hyperemesis Syndrome Presenting With Spontaneous Pneumomediastinum. Prim Care Companion CNS Disord 2020;22:19102509.

8. Weissberg D, Weissberg D. Spontaneous mediastinal emphysema. Eur J Cardiothorac Surg 2004;26:885-8.

9. Ryoo JY. Clinical analysis of spontaneous 
pneumomediastinum. Tuberc Respir Dis (Seoul) 2012;73:169-73.

10. Abolnik I, Lossos IS, Breuer R. Spontaneous pneumomediastinum. A report of 25 cases. Chest 1991;100:93-5.

11. Kaneki T, Kubo K, Kawashima A, et al. Spontaneous pneumomediastinum in 33 patients: yield of chest computed tomography for the diagnosis of the mild type. Respiration 2000;67:408-11.

12. Gerazounis M, Athanassiadi K, Kalantzi N, et al. Spontaneous pneumomediastinum: a rare benign entity. J Thorac Cardiovasc Surg 2003;126:774-6.

13. Jougon JB, Ballester M, Delcambre F, et al. Assessment of spontaneous pneumomediastinum: experience with 12 patients. Ann Thorac Surg 2003;75:1711-4.

14. Koullias GJ, Korkolis DP, Wang XJ, et al. Current assessment and management of spontaneous pneumomediastinum: experience in 24 adult patients. Eur J Cardiothorac Surg 2004;25:852-5.

15. Campillo-Soto A, Coll-Salinas A, Soria-Aledo V, et al. Spontaneous pneumomediastinum: descriptive study of our experience with 36 cases. Arch Bronconeumol 2005;41:528-31.

16. Freixinet J, García F, Rodríguez PM, et al. Spontaneous pneumomediastinum long-term follow-up. Respir Med 2005;99:1160-3.

17. Macia I, Moya J, Ramos R, Morera R, et al. Spontaneous pneumomediastinum: 41 cases. Eur J Cardiothorac Surg 2007;31:1110-4.

18. Mondello B, Pavia R, Ruggeri P, et al. Spontaneous pneumomediastinum: experience in 18 adult patients. Lung 2007;185:9-14.

19. Caceres M, Ali SZ, Braud R, Weiman D, et al. Spontaneous pneumomediastinum: a comparative study and review of the literature. Ann Thorac Surg 2008;86:962-6.

20. Al-Mufarrej F, Badar J, Gharagozloo F, et al. Spontaneous pneumomediastinum: diagnostic and therapeutic interventions. J Cardiothorac Surg 2008;3:59.

21. Takada K, Matsumoto S, Hiramatsu T, Kojima E, Watanabe H, Sizu M, Okachi S, Ninomiya K. Management of spontaneous pneumomediastinum based on clinical experience of 25 cases. Respir Med 2008;102:1329-34.

22. Perna V, Vilà E, Guelbenzu JJ, Amat I.

Pneumomediastinum: is this really a benign entity? When it can be considered as spontaneous? Our experience in 47 adult patients. Eur J Cardiothorac Surg 2010;37:573-5.

23. Okada M, Adachi H, Shibuya Y, et al. Diagnosis and treatment of patients with spontaneous pneumomediastinum. Respir Investig 2014;52:36-40.

24. Bakhos CT, Pupovac SS, Ata A, et al. Spontaneous pneumomediastinum: an extensive workup is not required. J Am Coll Surg 2014;219:713-7.

25. Ebina $M$, Inoue $A$, Takaba $A$, et al. Management of spontaneous pneumomediastinum: Are hospitalization and prophylactic antibiotics needed? Am J Emerg Med 2017;35:1150-3.

26. Langwieler TE, Steffani KD, Bogoevski DP, et al. Spontaneous pneumomediastinum. Ann Thorac Surg 2004;78:711-3.

27. Hasimoto CN, Cataneo C, Eldib R, et al. Efficacy of surgical versus conservative treatment in esophageal perforation: a systematic review of case series studies. Acta Cir Bras 2013;28:266-71.

28. Swanson JO, Levine MS, Redfern RO, Rubesin SE. Usefulness of high-density barium for detection of leaks after esophagogastrectomy, total gastrectomy, and total laryngectomy. AJR Am J Roentgenol 2003;181:415-20.

29. Wei CJ, Levenson RB, Lee KS. Diagnostic Utility of CT and Fluoroscopic Esophagography for Suspected Esophageal Perforation in the Emergency Department. AJR Am J Roentgenol 2020;215:631-8.
Cite this article as: Morgan CT, Maloney JD, Decamp MM, McCarthy DP. A narrative review of primary spontaneous pneumomediastinum: a poorly understood and resourceintensive problem. J Thorac Dis 2021;13(6):3721-3730. doi: $10.21037 /$ jtd-21-193 
Supplementary

Table S1 Vitals of primary spontaneous pneumomediastinum patients

\begin{tabular}{|c|c|c|c|c|c|}
\hline Study & Fever & Tachycardia & Hypotension & Tachypnea & Hypoxemia \\
\hline Abolnik et al. 1991 & NR & NR & NR & NR & NR \\
\hline Kaneki et al. 2000 & NR & NR & NR & NR & NR \\
\hline Gerazounis et al. 2003 & NR & NR & NR & NR & NR \\
\hline Jougon et al. 2003 & NR & 0 & 0 & NR & NR \\
\hline Koullias et al. 2004 & NR & NR & NR & NR & NR \\
\hline Weissberg \& Weissberg 2004 & 32 & 7 & NR & NR & NR \\
\hline Campillo-soto et al. 2005 & NR & 5.6 & NR & 5.6 & NR \\
\hline Newcomb \& Clarke 2005 & NR & NR & NR & NR & NR \\
\hline Freixinet et al. 2005 & NR & NR & NR & NR & NR \\
\hline Macia et al. 2007 & 14.6 & NR & NR & NR & NR \\
\hline Mondello et al. 2007 & 22 & NR & NR & NR & $33^{* *}$ \\
\hline Caceres et al. 2008 & NR & NR & NR & NR & NR \\
\hline Al-Mufarrej et al. 2008 & 5.9 & NR & NR & NR & NR \\
\hline Takada et al. 2008 & $28^{*}$ & NR & NR & NR & NR \\
\hline Perna et al. 2010 & NR & NR & NR & NR & NR \\
\hline Ryoo 2012 & NR & NR & NR & NR & NR \\
\hline Okada et al. 2014 & 10 & NR & NR & NR & NR \\
\hline Bakhos et al. 2014 & NR & NR & NR & NR & NR \\
\hline Ebina et al. 2017 & NR & NR & NR & NR & NR \\
\hline $\operatorname{AVE} \pm \operatorname{std} \operatorname{dev} \%[\mathrm{n}]$ & $18.8 \% \pm 10.3 \%[6]$ & $4.2 \% \pm 3.7 \%[3]$ & $0[1]$ & $5.6[1]$ & $33[1]$ \\
\hline
\end{tabular}

${ }^{*}$, fever defined as temperature $>37.2^{\circ} \mathrm{C}$; ${ }^{* *}$, mild hypoxemia. 
Table S2 Leukocytosis and WBC

\begin{tabular}{|c|c|c|}
\hline Study & $\begin{array}{c}\text { Leukocytosis } \\
(\%)\end{array}$ & $W B C \times 10^{3}$ \\
\hline Abolnik et al. 1991 & NR & NR \\
\hline Kaneki et al. 2000 & NR & NR \\
\hline Gerazounis et al. 2003 & NR & NR \\
\hline Jougon et al. 2003 & NR & NR \\
\hline Koullias et al. 2004 & NR & NR \\
\hline Weissberg \& Weissberg 2004 & 23 & NR \\
\hline Campillo-soto et al. 2005 & 5.6 & NR \\
\hline Newcomb \& Clarke 2005 & NR & NR \\
\hline Freixinet et al. 2005 & NR & NR \\
\hline Macia et al. 2007 & 42 & NR \\
\hline Mondello et al. 2007 & NR & NR \\
\hline Caceres et al. 2008 & 39 & NR \\
\hline Al-Mufarrej et al. 2008 & 5.9 & 9.42 \\
\hline Takada et al. 2008 & 56 & 10.1 \\
\hline Perna et al. 2010 & NR & NR \\
\hline Ryoo 2012 & 44 & 10.03 \\
\hline Okada et al. 2014 & NR & 11.97 \\
\hline Bakhos et al. 2014 & NR & NR \\
\hline Ebina et al. 2017 & NR & NR \\
\hline $\operatorname{AVE} \pm$ std dev [n] & $30.8 \pm 19.7[7]$ & WBC $10.4 \pm 1.1[4]$ \\
\hline
\end{tabular}

Table S3 Absence of effusions in spontaneous pneumomediastinum

\begin{tabular}{lc}
\hline Study & Effusion (\%) \\
\hline Abolnik et al. 1991 & $\mathrm{NR}$ \\
Kaneki et al. 2000 & $\mathrm{NR}$ \\
Gerazounis et al. 2003 & $\mathrm{NR}$ \\
Jougon et al. 2003 & $\mathrm{NR}$ \\
Koullias et al. 2004 & $\mathrm{NR}$ \\
Weissberg \& Weissberg 2004 & $\mathrm{NR}$ \\
Campillo-soto et al. 2005 & $\mathrm{NR}$ \\
Newcomb \& Clarke 2005 & $\mathrm{NR}$ \\
Freixinet et al. 2005 & $\mathrm{NR}$ \\
Macia et al. 2007 & $\mathrm{NR}$ \\
Mondello et al. 2007 & $\mathrm{NR}$ \\
Caceres et al. 2008 & 0 \\
Al-Mufarrej et al. 2008 & $\mathrm{NR}$ \\
Takada et al. 2008 & $\mathrm{NR}$ \\
Perna et al. 2010 & $\mathrm{NR}$ \\
Ryoo 2012 & $\mathrm{NR}$ \\
Okada et al. 2014 & 0 \\
Bakhos et al. 2014 & \\
Ebina et al. 2017 & $\mathrm{NR}$ \\
Percent effusions (number of studies) & \\
\hline & \\
\hline
\end{tabular}


Table S4 Management of concurrent pneumothorax

\begin{tabular}{|c|c|c|c|}
\hline Study & Thoracostomies & Pneumothoraces & Pneumothorax treated with chest tube (\%) \\
\hline Abolnik et al. 1991 & 1 & 3 & 33 \\
\hline Kaneki et al. 2000 & NR & NR & NR \\
\hline Gerazounis et al. 2003 & NR & NR & NR \\
\hline Jougon et al. 2003 & NR & NR & NR \\
\hline Weissberg \& Weissberg 2004 & 6 & 6 & 100 \\
\hline Campillo-soto et al. 2005 & NR & NR & NR \\
\hline Newcomb \& Clarke 2005 & 1 & 1 & 100 \\
\hline Freixinet et al. 2005 & 1 & 2 & 50 \\
\hline Al-Mufarrej et al. 2008 & NR & NR & NR \\
\hline Takada et al. 2008 & 0 & 0 & NA \\
\hline Perna et al. 2010 & NR & NR & NR \\
\hline Ryoo 2012 & NR & NR & NR \\
\hline Okada et al. 2014 & NR & NR & NR \\
\hline Bakhos et al. 2014 & NR & NR & NR \\
\hline Ebina et al. 2017 & NR & NR & NR \\
\hline
\end{tabular}


Table S5 Primary spontaneous pneumomediastinum recurrence

\begin{tabular}{|c|c|c|}
\hline Study & Recurrence rate (\%) & Follow up period (mos) \\
\hline Kaneki et al. 2000 & 0 & NR \\
\hline Gerazounis et al. 2003 & 4.5 & $36-144$ \\
\hline Jougon et al. 2003 & 0 & 3-60 (mean 19) \\
\hline Weissberg \& Weissberg 2004 & 0 & 12 \\
\hline Campillo-soto et al. 2005 & NR & NA \\
\hline Newcomb \& Clarke 2005 & 0 & NR \\
\hline Freixinet et al. 2005 & $0^{*}$ & $12-228$ \\
\hline Caceres et al. 2008 & 0 & $12-120$ \\
\hline Al-Mufarrej et al. 2008 & 0 & 0-34 (mean 6.72) \\
\hline Takada et al. 2008 & 0 & 22 \\
\hline Perna et al. 2010 & NR & NA \\
\hline Ryoo 2012 & NR & NA \\
\hline Okada et al. 2014 & 0 & 0.5 \\
\hline Bakhos et al. 2014 & 2 & $24-84$ \\
\hline Ebina et al. 2017 & 2.9 & NR \\
\hline
\end{tabular}

*, 10 of the 32 patients (31.2\%) were lost to follow up. 